\title{
Explorar la Construcción de la Identidad Docente en Profesionales de la Salud: Diseño y Validación de Instrumento
}

\author{
Catherine, R. Jara ${ }^{(1) \star}$ y Cristina Mayor-Ruiz ${ }^{(2)}$ \\ (1) Facultad de Ciencias de la Salud, Departamento Biomédico, Universidad de Antofagasta, \\ Avda. Angamos 601, Antofagasta-Chile (e-mail: catherine.jara@uantof.cl) \\ (2) Facultad de Educación, Departamento Didáctica y Organización Educativa, Universidad de Sevilla, \\ c/Pirotecnia s/n, Sevilla-España (e-mail: crismayr@us.es) \\ * Autor a quien debe ser dirigida la correspondencia
}

Recibido May. 8, 2018; Aceptado Jul. 11, 2018; Versión final Sep. 25, 2018, Publicado Feb. 2019

\begin{abstract}
Resumen
El objetivo de esta investigación es diseñar y validar un instrumento para explorar la construcción de la identidad docente en profesionales de la salud. El diseño del instrumento se estableció según dimensiones establecidas por el marco teórico. La validación del contenido se efectuó por juicio de expertos y de constructo con Alfa de Cronbach y Análisis factorial. La muestra la formaron 25 docentes de las Facultades de salud de la Universidad de Antofagasta y de la Universidad de Sevilla. En los resultados se obtuvo un cuestionario con cuatro dimensiones: Formación, Reflexión del proceso, Desempeño profesional y Elementos afectivos de la Identidad. Se obtuvo un alfa de Cronbach superior en todos los casos a 0,72 y un factor Kaiser-Meyer-Olkin (KMO) entre 0,72 y 0,5. En conclusión, el instrumento presenta una adecuada validez de contenido y constructo, lo que permite utilizarlo en estudios relacionados con construcción de identidad docente.
\end{abstract}

Palabras clave: desarrollo profesional; identidad profesional docente; área salud; profesorado universitario; validación instrumento.

\section{To Explore the Construction of Teaching Identity in Health Professionals: Design and Validation of Instrument}

\begin{abstract}
The objective of this investigation is to design and validate an instrument to explore the construction of the teaching identity in health professionals. The design of the instrument is established according to dimensions established by the theoretical frame. The validation of the content is carried out by expert's judgment and the construct by using Cronbach's Alpha and Factorial analysis. The sample was developed with 25 teachers from the health Faculties who belongs to the university of Antofagasta and the University of Sevilla. In the results it gotten a questionnaire with four dimensions: Training, Reflection of the process, Professional performance and Affective elements of the Identity with a Cronbach's alpha high in all the cases to 0,772 , and a Kaiser-Meyer-Olkin factor (KMO) between 0,72 and 0,5. In conclusion the instrument presents an adequate validity of content and construct, which allows to be used in studies related with the construction of the teaching identity.
\end{abstract}

Keywords: professional development; teacher professional identity; health area; university teaching staff; instrument validation. 


\section{INTRODUCCION}

Las carreras de la salud en los últimos años han presentado una gran demanda por parte de los estudiantes, por lo tanto las Universidades se han visto en la obligación de aumentar su planta académica contratando una mayor cantidad de profesionales de la salud expertos en su área profesional, que deben adoptar la posición de docente universitario a pesar de no tener la formación pedagógica pero sí la experiencia profesional que su carrera le ha otorgado. Siendo así, estos profesionales se insertan en ésta nueva profesión y deben lograr construir una identidad, ahora como docente del área de la salud con muchos dilemas e inseguridades para adaptarse a una cultura diferente. Por ello, surge la necesidad de obtener información sobre cuáles son los factores que influyen en la construcción de la identidad docente en los profesionales de la salud para permitir a las instituciones de educación superior implementar acciones tendientes a favorecer éste proceso y lograr mejorar constantemente su planta académica con una mirada hacia la excelencia del proceso de enseñanza-aprendizaje.

Bolivar et al. (2005) afirman que la identidad es la socialización de la persona que permite que la imagen del ser se configure bajo el reconocimiento de los otros. En este sentido, Stetsenko y Arievitch (2004) lo describen como el proceso de interacción entre las personas que contribuye a crear nuevos conocimientos y dar forma a identidades, a cambiar conductas y establecer creencias. Lo anterior, nos permite concluir que la identidad es un proceso dinámico que ocurre en el transcurso del tiempo, que puede interpretarse y reinterpretarse de acuerdo a las situaciones vividas por el sujeto y las relaciones con los demás.

\section{Construcción de la Identidad profesional docente}

La Identidad profesional es definida por Vloet y Van Swet (2010) como la importancia que le entrega el individuo a su quehacer. Beijaard et al. (2004) han implementado el término "agencia" para definir la identidad profesional. En éste mismo contexto Day (2006) señala que agencia es la capacidad del sujeto para lograr sus objetivos más valorados, donde existen factores históricos, sociológicos, psicológicos y culturales que permiten influenciar en su construcción. Lograr la profesionalización de su labor constituye el desarrollo de la construcción de la identidad profesional, las competencias, la formación, la experiencia profesional y los procesos de medición de desempeño profesional (Tejada, 2013).

Este artículo ha privilegiado la posición de la teoría de la valoración y el método de autoconfrontación de Hermans y Hermans-Jansen (1995), para construir un instrumento que permita al docente de la salud entregar un valor a los eventos significativos que influyen en la construcción de la identidad docente. Siguiendo en ésta línea Kelchtermans (2007) contribuye señalando que para lograr éste proceso es necesario que el docente atraviese momento críticos en el proceso de enseñanza-aprendizaje, debido a que la identidad profesional debe ser construida tanto en el aula como docente, pero también en privado en una autocomprensión profesional lo que permite al docente realizar una práctica reflexiva como un proceso a través del cual tiene acceso a conocerse como profesional, de manera que la experiencia pueda ser integrada y usada de manera estratégica en el proceso de enseñanza lo que conlleva al enriquecimiento, comprensión y modificación de las tareas realizadas como docente, como así sus creencias sobre la educación y sus teorías educativas subjetivas. Schwartzman et al. (2013) nos describen tres elementos claves de la práctica reflexiva; a) el acompañamiento a las tareas de enseñanza que desarrollan; b) la promoción de espacios de reflexión que permitan constantemente revisar la práctica; e c) incentivar la generación de nuevas propuestas de mejoramiento del proceso. Esto hace que el docente constantemente revise su práctica pedagógica, donde la interpretación y reinterpretación de las experiencias constituyen el pilar fundamental del desarrollo de la identidad.

En relación a lo anterior, el modelo de Hermans y Hermans-Jansen (1995), nos señala que el sujeto espera el mejoramiento contínuo en el proceso de enseñanza, autodesarrollarse, autoexigirse, permitir buscar herramientas para hacer cambios en sus roles y adquirir más agencias en sus vidas personales y profesionales como motivos intrínsecos para la construcción de la identidad docente, pero también el profesional necesita sentirse parte de una organización, de una cultura organizacional que le ofrezca las mejores condiciones para laborar, sentir el apoyo para mantener una identidad coherente y lidiar con los cambios que constantemente se dan en la educación (Doecke et al., 2000). De acuerdo a lo anterior, podemos analizar tres niveles que permiten la construcción profesional docente, el primero de ellos es el que involucra a la posición del docente frente al proceso de enseñanza, donde la autoimagen, la autoestima, y el automejoramiento contínuo, permiten al docente buscar los elementos necesarios para lograr con éxito el proceso de enseñanza, el segundo determinado por las relaciones sociales y cómo éstas influyen en la identidad del sujeto que pueden ser cambiantes de acuerdo al o los contextos donde se involucre y por último el nivel cultural donde la organización universitaria presenta un valor importantísimo, debido a que es la encargada de satisfacer las demandas de los docentes influyendo en su motivación laboral y la perspectiva futura de su desarrollo docente, factores claves en el proceso de construcción de identidad profesional. 
La construcción de la identidad docente en los profesionales de la salud estará influenciado por la experiencia de su vida personal como profesional sanitario, Ligorio (2007) señala que las profesiones que tienen vínculos firmes y duraderos con sentimientos humanos pueden impactarse por una interacción con sujetos que son importantes en su quehacer y representan finalmente una fuente de inspiración, donde el compromiso adquirido con el entorno social nos permite disfrutar de la tarea realizada y construir nuestra identidad profesional, por lo tanto, es un proceso cambiante que estará determinado por características personales, experiencias personales, contextos profesionales incluyendo aspectos relacionados con las emociones de las personas (Beauchamp y Thomas, 2009; Beijaard et al., 2000). Bolivar et al. (2005) aporta un aspecto que vincula a cómo los docentes viven su trabajo y las condiciones de satisfacción e insatisfacción que se producen al realizar la tarea. Es así, que los valores, motivaciones personales y emociones están en juego cuando los docentes desarrollan sus clases (Jansz y Timmers, 2002). Un estudio de Badia et al. (2014) describe en tres bloques los sentimientos hacia la docencia: a) el primero asociado a la motivación por la docencia; b) el segundo por las relaciones sociales (alumnos y compañeros) y, finalmente, c) los sentimientos de orgullo/satisfacción/descontento frente a la acción docente. Siendo tres ejes fundamentales en los cuales se debe trabajar para lograr la construcción de la identidad docente.

\section{Formación docente}

La formación docente se encuentra estrechamente relacionada con la construcción de la identidad profesional debido a que es la etapa donde se adquieren los primeros conocimientos de lo que es su labor pedagógica y donde podrá experimentar las primeras experiencias que marcaran su identidad profesional.

La escasez o ausencia, en algunos contextos de experiencias en formación pedagógica en educadores de la salud representan una falencia es por ello deben comenzar por comprender el proceso de enseñanzaaprendizaje para posteriormente formarse como profesional de la docencia, porque están ejerciendo un rol que los responsabiliza de la construcción de los aprendizajes de los estudiantes. Esto nos indica Estrada et al. (2016) que el docente "no es el fin del aprendizaje, sino una herramienta más, ya que su responsabilidad se centra en enseñar a aprender" (pp.46). Para Freitas et al. (2016) la responsabilidad del docente va más allá del mero enseñar, ya que exponen que "además del dominio de los conocimientos básicos del área de experiencia profesional, el docente debería poseer el dominio pedagógico y conceptual relacionado al proceso de enseñanza-aprendizaje" (pp.438).

Lo anterior, se ve reflejado en lo que Beijaard et al. (2004) hacen referencia a que el docente debe presentar pericia pedagógica determinada como la responsabilidad del docente con el proceso, pericia en la materia determinada por el conocimiento y por la experiencia en su disciplina y pericia educativa donde señala el compromiso y calidad con la educación que entrega. Finalmente, para lograr la formación el docente Cuadra y Catalán (2016) nos describen que debe pasar por "una serie de experiencias formales e informales de aprendizaje, ocurridas durante toda la vida" (pp. 302). Donde estas oportunidades que se le presentan le permitirán tener una visión más amplia del proceso de enseñanza (Batchelor y Scott, 2017) el cual en la formación de los profesionales de la salud es importante debido a que requiere actualización donde la investigación será primordial para adquirir nuevos conocimientos, permitiendo aplicar técnicas clínicas acordes a los avances tecnológicos (Barbera et al., 2015). Díaz (2016) describe que la investigación y la docencia están estrechamente relacionadas, porque el rol de investigador permite nutrir el de docente. Otro aspecto relevante de la formación es la gestión que entrega herramientas al profesor para actuar con confianza frente a situaciones administrativas en el ámbito universitario. Los planteamientos descritos describen la formación de un docente integral, poseedor de conocimientos pedagógicos, disciplinares, de gestión e investigación.

Tejada (2013) describe que la formación docente debe estar establecida por los siguientes aspectos: a) el académico pueda ingresar a un programa efectivo de formación durante sus primeros tres años en la profesión, b) que sean acompañados por profesores experimentados y c) que fueran escuchadas y satisfechas sus necesidades de formación por la institución en la cual laboran. En el caso de los docentes de la salud el primer aspecto resulta clave debido a que le permite comprender el proceso de enseñanzaaprendizaje a través de una formación establecida por la Institución que permita relacionar los aspectos pedagógicos con la experiencia disciplinar, como así permitir un acercamiento a la investigación. El segundo aspecto contribuye a que el docente pueda nutrirse de la experiencia de otros académicos y tener el apoyo necesario en sus primeras vivencias dentro de la academia universitaria; y finalmente el tercer aspecto es necesario para motivar al docente a seguir desarrollándose y construir su identidad profesional docente.

\section{OBJETIVO DEL ESTUDIO}

El objetivo que presentamos en este trabajo es conocer los factores que ayudan a construir la identidad profesional del docente de la salud desde las miradas pedagógicas, sociales, emocionales e institucionales. Los estudios realizados en éste ámbito son principalmente cualitativos basados en historias de vida del 
sujeto, entrevistas, o diarios que vienen a profundizar en los elementos más contextuales y/o personales sin posibilidades de extrapolar los resultados a poblaciones más amplias. En cambio, en ésta investigación se pretende diseñar y validar un instrumento cuantitativo que permita analizar grupos más amplios de sujetos y comparar así situaciones concretas derivadas de las distintas especialidades profesionales o de instituciones con características diferentes. Por lo tanto, presenta un mejor acceso a la población que se pretende estudiar y permite una respuesta rápida por parte de los profesionales que se le aplica el instrumento.

\section{METODOLOGIA}

La metodología utilizada en la investigación sobre los factores que influyen en la construcción de la identidad docente de los profesionales de la salud, es de tipo descriptivo desde una perspectiva básicamente cuantitativa ya que ello nos va a permitir buscar explicaciones más generales a fenómenos sociales, personales, profesionales y formativos de acuerdo a dimensiones determinadas por la literatura e investigaciones previas, donde el análisis estará enfocado en las respuestas que entregan los sujetos y representará una invitación a una discusión más profunda sobre el proceso de construcción de la identidad docente. En este trabajo nos centramos en presentar la parte de la metodología cuantitativa de la investigación, donde se muestran en la Fig. 1 las fases de diseño, fiabilidad y validación de contenido y constructo del instrumento para así explorar la construcción de la identidad profesional del profesorado universitario del área de la salud.

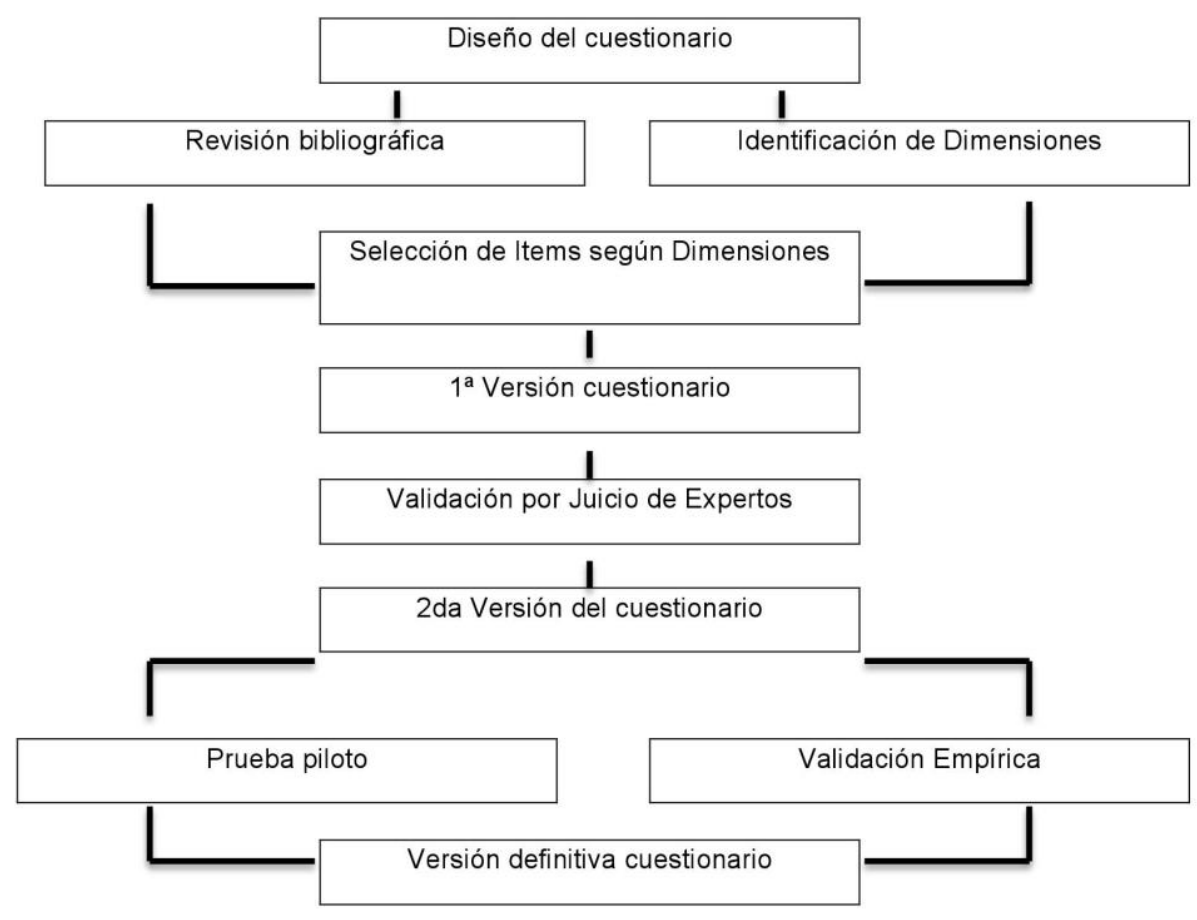

Fig.1: "Proceso de Diseño general del cuestionario"

Una vez realizada una revisión de la literatura existente en este campo se recogen las aportaciones de varios autores para determinar las dimensiones relacionadas con la construcción de la identidad docente, como los aspectos emocionales en sus experiencias de la labor docente; los sociales interpretadas por los vínculos entre profesorado principiante y estudiantes; los elementos dialécticos-pedagógicos como las tareas propias de la docencia y, finalmente, la dimensión institución-administrativa que involucra los elementos propios de la gestión (Gutiérrez et al., 2014). Soyago et al. (2008), en tanto le dan importancia a las dimensiones relacionadas con la experiencia práctica del docente, la sociabilización y apoyo entre pares y la actitud reflexiva que permiten reinterpretar la práctica docente. Tenti Fanfani (2005) aporta dimensiones relacionadas con las emociones donde incluyen aquellas relacionadas con dificultades experimentadas durante el proceso de enseñanza-aprendizaje, la satisfacción con el trabajo y el reconocimiento social, aunque también las relacionadas con la Institución, donde la formación y actualización representan un rol clave en la construcción de la identidad. Con toda ésta información se diseña una primera versión del cuestionario identificando inicialmente las dimensiones más significativas para el investigador.

1) Dimensión Formación: Esta dimensión es importante debido a que el profesional de la salud no tiene una formación académica y representa un área relevante que deben ser indagadas por su repercusión en la construcción de la identidad profesional. Así, Friesen y Besley (2013) comentan que los programas de 
formación tienen el potencial de facilitar o interferir en el desarrollo de la identidad en relación con el aprendizaje de los valores y normas asociadas a la profesión docente. Las variables de ésta dimensión son las relacionadas con la formación tanto como actividades formales organizadas por una Institución como la capacitación por cuenta propia del docente que le permiten adquirir conocimiento pedagógicos propios de la labor docente. 2) Dimensión Construcción de la Identidad docente: Está dimensión está determinada por elementos que influyen en la construcción de la identidad, como son las experiencias profesionales y docentes vividas por el sujeto y las relaciones sociales en las cuales ha estado inmerso. La primera se logra a través de la reflexión del quehacer ofreciéndole al profesional una mirada sobre las inquietudes, aciertos y desaciertos de su labor lo que conlleva a la rectificación de sus acciones y el compromiso que adquiere con la práctica. La segunda permite la reinterpretación de su identidad de acuerdo a las relaciones sociales que establezca. Las variables de ésta dimensión son las que permiten la reflexión sobre su práctica profesional y educativa orientándolo hacia un modelo de enseñanza, un sistema de relaciones y redes de apoyo que genera el docente con los pares y con los estudiantes. 3) Dimensión Elementos afectivos de la Identidad: Esta dimensión permite conocer los sentimientos y emociones con las que se va enfrentando el profesional de la salud al realizar la docencia, considerando la susceptibilidad emocional presente en su práctica pedagógica. Friesen y Besley (2013) infieren que la profesión docente debe hacer que el profesor experimente sus sentimientos con la labor realizada hasta encontrar las características que lo representen como miembro de la profesión académica. Por su parte, Day et al. (2005) incluyen la motivación por el trabajo y con las personas con que se trabaja. Las variables de ésta dimensión son las que tienen relación con motivación y/o desmotivación por el trabajo realizado, la valoración, apoyo y clima organizacional en el quehacer académico, etc. En ésta dimensión se han agregado cuatro preguntas de selección múltiple con indicadores considerados en la literatura como importantes para describir los períodos de reinterpretación de la identidad.

En todas las dimensiones las opciones de respuestas se ajustan a una escala de medición Likert ordinal (donde el 1 representa el nunca y el 5 siempre) y en cuatro de ellas a preguntas de opción múltiple (Tabla 1).

\section{Diseño del Cuestionario}

Una vez definidas las Dimensiones se elaboran los ítems en cada una de ellas, configurándose la primera versión del cuestionario (Tabla 1), el cual quedó conformado por 66 ítems distribuidos en una primera parte dedicada a la Identificación del académico (surgiendo dos versiones debido a las características administrativas de las dos Universidades participantes) y tres dimensiones: a) Formación, b) Construcción de la Identidad docente y c) Elementos afectivos de la Identidad. Esta primera versión fue enviada a la validación de contenido por juicio de expertos.

Tabla 1: Estructura de la primera versión del cuestionario

\begin{tabular}{|c|c|c|}
\hline Cuestionario & Preguntas múltiples & Preguntas Escala de Likert \\
\hline Identificación del académico & 11 & 10 \\
\hline Dimensión Formación & & 29 \\
\hline Dimensión Construcción de la Identidad docente & & 12 \\
\hline Dimensión Elementos afectivos de la Identidad & 4 & \multicolumn{2}{|c|}{} \\
\hline
\end{tabular}

\section{Validación del cuestionario por Juicio de Expertos}

El contenido del cuestionario se valida a través de juicios de expertos, los cuales fueron seleccionados de acuerdo a los siguientes criterios: a) Doctor y b) Especialista en formación inicial, identidad profesional o formación del profesorado. El instrumento se entregó personalmente o se envió través de Google Drive a doctores en CC.Educación, cuatro de la Universidad de Sevilla (España), dos de la Universidad de Antofagasta (Chile), uno de la Universidad Minas Gerais (Brasil) y uno de la Universidad de Huelva (España). Las aportaciones de estos expertos nos llevó a considerar que la dimensión "Construcción de la identidad docente" debía ser separada en dos dimensiones: "Reflexión del proceso" y "Desempeño profesional", en la cual en la primera las variables que se consideran serán las relacionadas con el proceso de enseñanza-aprendizaje, vivencias del docente en éste período y la reflexión del profesor al término de éste. En la segunda Dimensión "Desempeño profesional", las variables están enfocadas en las relaciones sociales y acciones dentro de su labor consideradas importantes para un docente universitario, como son las relacionadas con proyectos, publicaciones y gestión tanto de la investigación como de la docencia. En ambas dimensiones las opciones de respuestas se ajustan a una escala de medición Likert ordinal (donde el 1 representa el nunca y el 5 siempre). 
Se agregan preguntas abiertas sugeridas por los jueces para comprobar si las dimensiones consideradas por el investigador son las importantes para los profesores de la salud para construir su identidad profesional docente. En la dimensión "Reflexión del proceso" se agrega la pregunta relacionada con la distribución del tiempo de dedicación al quehacer académico, en la dimensión "Desempeño profesional" la pregunta tiene relación con el desarrollo profesional que puede lograr el docente con su práctica pedagógica y finalmente en la dimensión "Elementos afectivos de identidad" la pregunta es cuáles son las características particulares que debe presentar un docente de salud. Estas preguntas se trabajan mediante una distribución de frecuencia porcentual, que permite asignar un porcentaje a la respuesta del docente frente a la pregunta abierta para posteriormente agruparlas cuantitativamente según grado de importancia determinado por los sujetos (Bisquerra, 2014) corroborando si los profesionales de la salud concuerdan con los factores que influyen en la construcción de la identidad docente descritos en la literatura y tomados en consideración en ésta investigación. Finalmente, todo lo anterior nos permitió diseñar la segunda versión del instrumento (Tabla 2), el cual quedó conformado por 52 ítems distribuidos en una sección dedicada a la Identificación del académico y cuatro Dimensiones: a) D. Formación, b) D. Reflexión del proceso, c) D. Desempeño profesional, y d) D. Elementos afectivos de la Identidad. Sobre esta versión se procede a realizar la prueba piloto.

Tabla 2: Estructura de la segunda versión del cuestionario

\begin{tabular}{|l|c|c|c|}
\hline \multicolumn{1}{|c|}{ Cuestionario } & $\begin{array}{c}\text { Preguntas } \\
\text { múltiples }\end{array}$ & $\begin{array}{c}\text { Preguntas Escala de } \\
\text { Likert }\end{array}$ & $\begin{array}{c}\text { Preguntas } \\
\text { Abiertas }\end{array}$ \\
\hline Identificación del académico & 11 & & \\
\hline Dimensión Formación & & 7 & 1 \\
\hline Dimensión Reflexión del proceso & & 10 & 1 \\
\hline Dimensión Desempeño profesional & & 11 & 1 \\
\hline $\begin{array}{l}\text { Dimensión Elementos afectivos de la } \\
\text { identidad }\end{array}$ & 4 & 6 & \\
\hline
\end{tabular}

\section{Aplicación de la Prueba piloto del cuestionario}

La prueba piloto se realizó a los docentes de la Facultad de Ciencias de la Salud de la Universidad de Antofagasta y de las Facultades del área de la salud de la Universidad de Sevilla. El objetivo de esta prueba es determinar la comprensión, la aceptabilidad y el tiempo de aplicación del instrumento. Bisquerra (2014) señala que se puede realizar una prueba piloto a menor escala con todos los elementos que se utilizarán en el cuestionario final, donde los sujetos participantes en el piloto están representados por diferentes profesionales que tienen los criterios principales de la población a estudiar. A partir de ésta experiencia previa Bisquerra (2014) establece que se pueden comenzar a realizar estudios de fiabilidad y validez del instrumento mediante pruebas estadísticas. En éste estudio se utilizó el programa computacional SPSS versión 21.

\section{Descripción de la muestra de la prueba piloto}

El cuestionario en la prueba piloto fue aplicado a una muestra no probabilística por criterio: ser docente del área de la salud, tener cinco años de experiencia docente y tener experiencia profesional en su área disciplinar.

La muestra $(n=25)$ se distribuyó a diferentes profesionales de las Facultades de Ciencias de la Salud de la Universidad de Antofagasta $(n=10)$ y de las Facultades del área de la Salud de la Universidad de Sevilla $(n=15)$.

La edad de los docentes de la Facultades del área de la Salud de Universidad de Sevilla está distribuida entre los 41 y 60 años. En cambio, en la Facultad de la Salud de la Universidad de Antofagasta el promedio va desde los 31 a 50 años. El 60\% de mujeres contestaron la encuesta en la Universidad de Antofagasta, en cambio en la Universidad de Sevilla hubo una mayor concentración en los varones, con un 53,3\%. En relación a los títulos profesionales podemos indicar que existen diferentes titulaciones entre las dos Universidades, debido a que la Universidad de Antofagasta no imparte la carrera de farmacia, ni la de podología (ya que en la legislación chilena es un título técnico no universitario) y la de fisioterapia (que es una especialidad dentro de la kinesiología). Por otro lado, hay convergencias entre las dos Universidades como el Grado de Enfermería, de Medicina y el de Matrones (que inicialmente se formaron como enfermeros). En cambio, la Universidad de Sevilla no imparte las titulaciones de Tecnólogo médico, de Nutricionista, ni de Fonoaudiólogo. En el caso del grado académico se presenta un mayor número de doctores en la Universidad de Sevilla, ya que se alcanza un 64,29\%; en cambio en la Universidad de 
Antofagasta predomina el grado de magister con un $90 \%$. Un $60 \%$ de los docentes de la Universidad de Antofagasta responden que poseen una formación específica en Educación a través de Magister. En la Universidad de Sevilla no se poseen datos al respecto. El tipo de contrato que se presenta entre las dos Universidades administrativamente es diferente debido a que en la Universidad europea se establece por concursos, ya sea de méritos $26,67 \%$ o de oposición $33,33 \%$, o bien por otras vías (40\%), en cambio en la Universidad chilena el contrato tiene relación con el vínculo que el académico tiene con la institución; así se distribuye entre jornada completa/propiedad, en un 30\%, jornada completa/contrata con un $60 \%$ y un $10 \%$ a honorarios.

\section{RESULTADOS DE LA PRUEBA PILOTO}

Después de realizar la prueba piloto, los académicos realizan algunas observaciones que se consideraron para mejorar la comprensión de las dimensiones. El tiempo de respuesta del cuestionario fue de 12-15 minutos, lo que representa un tiempo adecuado para que el docente de la salud pueda terminarlo completo y sin interrupciones.

Se realiza una matriz de datos con todas las respuestas de los académicos que participan en esta prueba piloto para posteriormente aplicar el programa estadístico SPSS versión 21. Las pruebas estadísticas que se realizan son las siguientes: a) Alfa de Cronbach: la cual nos permite determinar la fiabilidad del instrumento estableciendo medidas de coherencia interna entre los coeficientes. Ellos deben oscilar entre 0 , que significa nula confiabilidad, y 1 , que representa confiabilidad total. Podemos decir que si el coeficiente supera el 0.70 es aceptable para la fiabilidad del cuestionario, siendo además más fiables utilizando una potencia estadística del 100\% y una seguridad del 95\% (Bisquerra, 2014), b) Análisis factorial: Kaiser Meyer Olkin (KMO) y Prueba de esfericidad de Bartlett. El análisis factorial según Morrison (2003) se realiza para determinar la válidez de constructo, por tanto, el objetivo es definir la estructura subyacente de un conjunto de datos analizando las interrelaciones entre los ítems del instrumento. Este análisis se realizó a través de las pruebas de Kaiser-Meyer-Olkin (KMO) para comprobar si las correlaciones parciales entre los ítems son los suficientemente pequeñas para que el cuestionario pueda ser aplicado (debiendo ser $p$ mayor de 0,5) y la prueba de esfericidad de Bartlett para comprobar la significación de los ítems en una matriz de correlaciones (debiendo ser $p$ menor a 0,005), éste criterio se utilizó para conservar o eliminar las variables de las dimensiones mencionadas.

Tabla 3: Análisis factorial de las dimensiones y fiabilidad del cuestionario

\begin{tabular}{|l|c|c|c|}
\hline \multicolumn{1}{|c|}{ Dimensión } & \multicolumn{2}{c|}{ Análisis factorial } & Fiabilidad \\
\hline & KMO & Bartlett & 0,779 \\
\hline Formación & 0,720 & 0,000 & 0,726 \\
\hline Reflexión del Proceso & 0,519 & 0,000 & 0,728 \\
\hline Desempeño profesional & 0,590 & 0,004 & 0,724 \\
\hline Elementos afectivos de la Identidad & 0,503 & 0,001 & Cronbach \\
\hline
\end{tabular}

En la Tabla 3 se puede observar la consistencia interna del instrumento determinada por la fiabilidad a través del Alfa de Cronbach para cada una de las dimensiones. Como se puede observar, todos los casos se notan aparentemente bajos pero se relacionan éstas cifras directamente con la cantidad y distribución de las personas evaluadas, de igual modo Bisquerra (2014) señala que si sus valores son superiores a 0.70 indican la confiabilidad del instrumento para ser aplicado. Es la dimensión "Formación" la que presenta el valor más alto (0.779) y la dimensión "Elementos afectivos de la Identidad" el más bajo (0.724). El cuestionario definitivo presenta un Alfa de Cronbach de 0.834 , para el total de las dimensiones del instrumento, lo que representa la confiabilidad del instrumento que estamos validando.

En las distintas pruebas del análisis factorial los valores obtenidos fueron los siguientes: la prueba de adecuación muestral de KMO presenta en todas las dimensiones valores superiores a 0.5 , lo cual indica que las correlaciones entre las variables son pequeñas y que el cuestionario puede ser aplicado. En el caso de la prueba de esfericidad de Bartlett todas las dimensiones se encuentran por debajo de 0.005 , lo que comprueba la significación de cada uno de los ítems en la matriz de correlaciones (Morrison, 2003).

Una vez realizadas ambas pruebas, de fiabilidad y factorial, se construye la versión definitiva del cuestionario (Tabla №4) que queda conformada por 51 ítems distribuidos en una sección dedicada a la Identificación del académico y cuatro dimensiones a) D. Formación b) D. Reflexión del proceso c) D. Desempeño profesional y d) D. Elementos afectivos de la Identidad. 
Tabla 4: Estructura de la versión definitiva del cuestionario

\begin{tabular}{|l|c|c|c|}
\hline \multicolumn{1}{|c|}{ Cuestionario } & $\begin{array}{c}\text { Preguntas } \\
\text { múltiples }\end{array}$ & $\begin{array}{c}\text { Preguntas Escala de } \\
\text { Likert }\end{array}$ & $\begin{array}{c}\text { Preguntas } \\
\text { Abiertas }\end{array}$ \\
\hline Identificación del académico & 11 & & \\
\hline Dimensión Formación & & 10 & 1 \\
\hline Dimensión Reflexión del proceso & & 11 & 1 \\
\hline Dimensión Desempeño profesional & & 6 & 1 \\
\hline Dimensión Elementos afectivos de la identidad & 4 & & \\
\hline
\end{tabular}

\section{Preguntas abiertas del cuestionario}

Como señalamos anteriormente, el juicio de experto sugirió la incorporación de preguntas abiertas para corroborar si las dimensiones del cuestionario se encuentran en relación a los aspectos considerados por los profesionales de la salud importantes para el proceso de construcción de la identidad docente. Entendemos que debe ser el sujeto el que aporte variadas respuestas procedentes de situaciones particulares que nos pueden ayudar a enriquecer las opciones de respuestas y que por tanto no están delimitadas a priori por el investigador. Es así que se consideran tres cuestiones: 1) Señale una definición de Ud. como docente ¿Cómo se considera? ¿Qué rasgos o características le definen?; 2) Siente que el trabajo que desempeña le ha permitido desarrollarse personal y profesionalmente durante éstos años ¿Por qué? ¿Qué le falta?; 3) A su juicio, ¿cómo debería distribuir el tiempo de dedicación a la docencia, investigación y gestión?

En base a las respuestas obtenidas se realizó una distribución de frecuencia porcentual, que permitió asignar un porcentaje a la respuesta del docente para posteriormente agruparlas cuantitativamente según grado de importancia determinado por los sujetos (Bisquerra, 2014) corroborando si los profesionales de la salud concuerdan con los factores que influyen en la construcción de la identidad docente descritos en la literatura y tomados en consideración en ésta investigación.

Tabla 5: Distribución de Frecuencia para la Pregunta "Señale una definición de Ud. como docente ¿Cómo se considera? ¿Qué rasgos o características le definen?”

\begin{tabular}{|l|c|c|c|}
\hline \multicolumn{1}{|c|}{ Atributo } & Frecuencia & Atributo & Frecuencia \\
\hline Compromiso & $18,6 \%$ & Facilitador Aprendizaje & $4,6 \%$ \\
\hline Responsabilidad & $16 \%$ & Reflexión del proceso & $4,6 \%$ \\
\hline Cercano al estudiante & 11,6 & Motivador & $4,6 \%$ \\
\hline Dominio tema & $9,3 \%$ & Perfeccionista & $2,3 \%$ \\
\hline Proactiva & $6,9 \%$ & Eficaz & $2,3 \%$ \\
\hline Exigente & $6,9 \%$ & Innovador & $2,3 \%$ \\
\hline Empatía & $6,9 \%$ & Dedicación a la Investigación & $2,3 \%$ \\
\hline
\end{tabular}

En la Tabla 5 podemos observar que los atributos "compromiso" y "responsabilidad" han sido considerados los aspectos más relevantes por los docentes al responder a la pregunta, las cuales representan características personales del profesional y la base de la identidad docente. Así también podemos destacar que un gran número de docentes se definen como "cercano al estudiante" y con "dominio del tema", esto se reafirma con lo que indica Beijaard et al. (2004) que hacen referencia a que el docente debe presentar pericia pedagógica, en la materia y experiencia en su disciplina. También hay que destacar aquellas respuestas que obtienen índices bajos y que están vinculados con la docencia como son "facilitador del aprendizaje", "motivador" e "innovador", según el marco teórico éstas características representan aspectos importantes para los docentes y que se encuentran asociados a nuevos modelos de enseñanza, pero en los profesores de la salud se puede visualizar que no son tan relevantes éstos atributos y todavía consideran la enseñanza tradicional como una práctica adecuada para el nivel universitario.

En la Tabla 6 podemos observar que el atributo "me encuentro desarrollado personal y profesionalmente como docente" ha sido considerado uno de los más sugeridos. Esto resulta importante para el proceso de construcción de la identidad ya que como Bolívar et al. (2005) señala si el docente se siente satisfecho con la tarea realizada se facilita la construcción de la identidad. En esta pregunta se profundiza en las áreas que este profesorado considera deficitarias como son la falta de formación docente y la falta de desarrollo de la 
investigación. Esto es corroborado por Friesen y Besley (2013) donde describe que la capacitación pedagógica es necesaria en los docentes universitarios en sus primeros años de ejercicio. La falta de desarrollo de la investigación académica y la de formación en gestión necesitan los recursos y el apoyo de la institución para poder llevarla a cabo.

Tabla 6: Distribución de Frecuencia para la Pregunta "Siente que el trabajo que desempeña le ha permitido desarrollarse personal y profesionalmente durante éstos años ¿Por qué? ¿Qué le falta?”

\begin{tabular}{|l|c|l|c|}
\hline \multicolumn{1}{|c|}{ Atributo } & Frecuencia & \multicolumn{1}{c|}{ Atributo } & Frecuencia \\
\hline $\begin{array}{l}\text { Me encuentro desarrollado personal y } \\
\text { profesionalmente como docente }\end{array}$ & $45,9 \%$ & Falta estabilidad laboral & 5,4 \\
\hline Falta capacitación en docencia & $10,81 \%$ & $\begin{array}{l}\text { La motivación para la docencia se } \\
\text { perjudica por el clima laboral }\end{array}$ & 5,4 \\
\hline Falta desarrollo en investigación & 10,8 & Faltan recursos para docencia e investigar & 5,4 \\
\hline Siento apoyo de las autoridades & $5,4 \%$ & Desarrollado en Docencia y Gestión & $5,4 \%$ \\
\hline Todavía desarrollándome & $5,4 \%$ & Falta capacitación en gestión & 2,7 \\
\hline Me gusta compartir experiencias & 5,4 & & \\
\hline
\end{tabular}

Tabla 7: Distribución de Frecuencia para la Pregunta "Cómo debería a su juicio distribuir el tiempo de dedicación a la docencia, investigación y gestión"

\begin{tabular}{|l|c|l|c|}
\hline \multicolumn{1}{|c|}{ Atributo } & Frecuencia & \multicolumn{1}{|c|}{ Atributo } & Frecuencia \\
\hline Docencia-Investigación y Gestión & $26,08 \%$ & Docencia y Gestión & $4,3 \%$ \\
\hline Docencia-Gestión e Investigación & $17,30 \%$ & Función principal la docencia & $4,3 \%$ \\
\hline Iguales proporciones & $13,04 \%$ & Gestión-Docencia e Investigación & $4,3 \%$ \\
\hline Muy difícil distribuir el tiempo & $13,04 \%$ & Investigación y Gestión & $4,3 \%$ \\
\hline $\begin{array}{l}\text { De acuerdo a los cargos de gestión que } \\
\text { ocupa }\end{array}$ & $8,69 \%$ & $\begin{array}{l}\text { Se necesita tiempo protegido para } \\
\text { investigar }\end{array}$ & $4,3 \%$ \\
\hline
\end{tabular}

En la Tabla 7 podemos observar que el atributo "docencia-investigación y gestión" ha sido considerado un aspecto relevante por más del $25 \%$ de los docentes, y en menor medida el que agrupa docencia-gestión e investigación. Esta pregunta nos ayuda a profundizar en la distribución del tiempo de los académicos y en cuáles serían los aspectos que considera como más relevantes en su quehacer profesional, señalando que, aún cuando las tres actividades (docencia, investigación y gestión) son importantes, es difícil tratar de desarrollarlas dentro de su actividad diaria debido a la cantidad de tareas que deben realizar. Esto reafirma lo que señala Díaz (2016) que la docencia es importante pero siempre acompañada por la investigación, debido a que son áreas que permiten al académico estar actualizado sobre los procedimientos clínicos que van surgiendo y que deben ser transmitidos a los estudiantes para que puedan ser aplicados en su futuro quehacer profesional.

Las preguntas abiertas nos han ayudado a reafirmar lo que señala la literatura sobre cuales son los aspectos relevantes en la construcción de la identidad profesional docente, como son los relacionados con el compromiso con la práctica pedagógica, el desarrollo profesional y la motivación del docente para continuar con el quehacer académico, pero también nos ha permitido conocer si existen diferencias entre los docentes de diferentes especialidades de la salud sobre cuáles son los factores considerados importantes para ellos y nos evidencia que temas relacionados con la formación docente es un elemento considerado como deficitario en su quehacer académico. Todo lo anterior nos permite corroborar el marco teórico de la investigación y presentarlo en la versión final del instrumento.

\section{DISCUSIÓN}

En la bibliografía consultada las investigaciones sobre la construcción de la identidad docente son mayoritariamente de tipo cualitativo lo que permite recoger experiencias idiosincráticas de situaciones vividas por los propios protagonistas que han hecho que estos profesionales pasen por momentos de cambios y reflexionen sobre su quehacer (Beauchamp y Thomas, 2009; Beijaard et al., 2000). En el caso de los profesionales de la salud, parece interesante conocer estos aspectos debido a que los procesos de formación juegan un rol fundamental permitiéndoles desarrollar y/o modificar su identidad profesional como docentes, normalmente compatibilizada con su identidad como profesional de la salud. Este cuestionario 
permite conocer los procesos de construcción de la identidad a partir de las dimensiones: Formación, Reflexión sobre el proceso, Desarrollo profesional y de los Elementos afectivos de la identidad que influyen en el docente, lo cual nos permite profundizar sobre todas las dimensiones implicadas. Un conocimiento exhaustivo sobre el profesorado de estas áreas permitirá que se puedan implementar en las instituciones universitarias acciones en apoyo al profesional que se dedica a la docencia, desde una mirada global del proceso.

El instrumento nos ha permitido profundizar sobre los aspectos que juegan un papel importante en la construcción de la identidad y con una mirada del académico que vive el proceso. Los temas afectivos, como la cercanía con el estudiante, son aspectos que favorecen su identidad, así como los aspectos relacionados con el compromiso y la responsabilidad, pero resalta el hecho de que los nuevos modelos pedagógicos no sean aspectos relevantes para el docente de la salud. Otro elemento importante es que la docencia deba ir ligada a la investigación (Díaz, 2016). Como bien lo describen algunos autores como Monereo y Dominguez (2014) los académicos enseñan mejor cuando investigan, algo que ha sido confirmado por los docentes consultados en este trabajo. La falta de capacitación es el aspecto más falente para el académico debido a que en su formación de profesional sanitario no contó con las competencias para impartir docencia y por ello es que deben continuamente perfeccionarse como lo confirman Friesen y Besley (2013) cuando apuntan que los programas de formación inicial tienen el potencial de facilitar o interferir con el desarrollo de la identidad.

\section{CONCLUSIONES}

Recurrimos ahora a los objetivos de este trabajo, cual era el diseño y validación de un cuestionario que nos ayudara a determinar la construcción de la identidad docente de profesionales de la salud. Una vez realizado el correspondiente juicio de expertos y prueba piloto con las pruebas de fiabilidad y análisis factorial, se pueden obtener las siguientes conclusiones:

a) El Cuestionario "La Construcción de la Identidad Profesional de docentes universitarios del área de la salud" es un instrumento de fácil y rápida administración lo cual nos ha permitido recoger una valiosa información entre los profesionales sanitarios que, por su función asistencial su tiempo es limitado, y por tanto es difícil acceder a ellos. Sin embargo, éste instrumento nos ha permitido llegar a una población elevada de docentes de la salud en un período breve de tiempo; b) La metodología cuantitativa utilizada presenta una adecuada validez y fiabilidad, comprobada a través de las correlaciones logradas entre ítem/total o entre ítem/ítem, según las pruebas estadísticas aplicadas c) Los resultados encontrados nos permiten explorar y validar estadísticamente cada dimensión sobre la construcción de la identidad docente del profesional de la salud y finalmente, d) La metodología realizada puede ser ampliada a otras muestras de diferentes profesionales que se dedican a la labor pedagógica, lo que permitirá a la institución conocer las distintas necesidades e implementar acciones que contribuyan a mejorar el proceso de enseñanzaaprendizaje de los estudiantes universitarios.

Finalmente podemos concluir afirmando que el Cuestionario para analizar "La Construcción de la Identidad Profesional de docentes universitarios del área de la salud" es un instrumento accesible y válido para utilizarlo en el campo de la investigación cuantitativa sobre la construcción de la identidad docente.

\section{REFERENCIAS}

Badia, A., C. Monereo y J. Meneses, Affective Dimension of University Professors about their Teaching: An Exploration through the Semantic Differential Technique, doi: 10.11144/Javeriana.UPSY13-1.adup, Universitas Psychologica, 13(1), 161-173 (2014)

Barbera, O., D. Cecagno, L. Seva., H. Crecencia, M. López y S. Maciá, Formación académica del profesional de enfermería y su adecuación al puesto de trabajo, doi: 10.1590/0104-1169.0432.2569, Revista Latinoamericana Enfermagem, 23(3), 404-410 (2015)

Batchelor, K. y A. Scott, Down the Rabbit Hole: Using The Matrix to Reflect on Teacher Education, doi: 10.1080/17425964.2017.1286577, Studying Teacher Education, 13(1), 68-86 (2017)

Beauchamp, C. y L. Thomas, Understanding teacher identity: An overview of issues in the literature and implications for teacher education, doi: 10.1080/03057640902902252, Cambridge Journal of Education, 3(2)9, 175-189 (2009)

Beijaard, D., N. Verloop y J. Vermunt, Teachers perceptions of professional identity: An exploratory study from a personal knowledge perspective, doi: 10.1016/50742-051X(00)00023-8, Teaching and Teacher Education, 16(7), 749764 (2000)

Beijaard, D., P.C. Meijer y N. Verloop, Reconsidering research on teachers' profesional identity, doi:10.1016/j.tate.2003.07.001, Teaching and Teacher Education, 20, 107-128 (2004)

Bisquerra, R.A., Metodología de la Investigación educativa, 4ª Ed., 320-365, Editorial La Muralla, Madrid, España (2014) 
Bolivar, B.A., C.M. Fernández y R.E. Molina, Investigar la identidad profesional del profesorado: Una triangulación secuencial, Forum: Qualitative social Research Sozialforschung, ISSN: 1438-5627, 6(1), 1-26 (2005)

Cuadra, M.D. y A.J. Catalán, Teorías subjetivas en profesores y su formación profesional, doi: 10.1590/5141324782016216517, Revista Brasileira de Educação, 21(65), 299-324 (2016)

Day, C., A. Kington, G. Stobart y P. Sammons, The personal and professional selves of teachers: stable and unstable identities, doi: 10.1080/01411920600775316, British Educational Research Journal, 32(4), $601-616$ (2006)

Day, C., B. Elliot y A. Kington, Reform, standards and teacher identity: Challenges of sustaining commitment, doi: 10.1016/j.tate.2005.03.001, Teaching and Teacher Education, 21(5), 563-577 (2005)

Díaz, M.W., Formación del profesorado universitario, evaluación de la actividad docente, recursos y promoción profesional, Estudios Pedagógicos, ISSN: 0716-050X, 42(1), 65-85 (2016)

Doecke, B., J. Brown y J. Loughran, Teacher talk: The role of story and anecdote in constructing profesional knowledge for beginning teachers, doi:org/10.1016/S0742-051X(99)00065-7, Teaching and Teacher Education, 16, 335-348 (2000)

Estrada, M., D. Monferrer y D. Moliner, El aprendizaje cooperativo y las habilidades socioemocionales: Una experiencia docente en la asignatura técnica de ventas, doi: 10.4067/S0718-50062016000600005, Revista Formación Universitaria, 9(6), 43-62 (2016)

Freitas, D., E. Santos, L. Lima., L. Miranda, E. Vasconcelos y P. Nagliate, Teachers' knowledge about teaching learning process and its importance for professional education in health, doi: 10.1590/1807-57622014.1177, Interface (Botucatu), 20(57), 437-448 (2016)

Friesen, M.D. y S.C. Besley, Teacher identity development in the first year of teacher education: A developmental and social psychological perspective, doi: 10.1016/j.tate.2013.06.005, Teaching and Teacher Education, 36, 23-32 (2013)

Hermans, H. y E. Hermans-Jansen, Self-narratives: The construction of meaning in psychotherapy, 1르. Ed., 31-73, The Guilford Press, New York, USA (1995)

Jansz, J. y M. Timmers, Emotional Dissonance. When the experience of an emotion jeopardizes and individual's identity, doi.org/10.1177/0959354302121005, Theory \& Pschology, 12(1), 79-95 (2002)

Kelchtermans, G., Capturing the multidimensionality of teacher professionalism: Broad and deep and reflection. In J. Van Swet, B. Smit \& P. Ponte (Eds.), Postgraduate programs as platform: A research-led Approach, 97-109, Sense Publishers, Rotterdam, Taipei (2007)

Ligorio, B.M. y L. Tateo, “Just for passion”: Dialogical and narrative construction of teachers' profesional identity and educational practices, European Journal of School Psychology, ISSN: 1723-7254, 5(2), 115-142 (2007)

Martín-Gutiérrez, A., J. Conde-Jiménez y C. Mayor-Ruiz, "La identidad profesional docente del profesorado novel universitario", doi.org/10.4995/redu.2014.5618, REDU Revista de Docencia Universitaria, 12(4), 141-160 (2014)

Monereo, C. y C. Domínguez, La identidad docente de los profesores universitarios competentes, doi: 105944/educxx1.17.211480, Educación XX1, 17(2), 83-104(2014)

Morrison, D.F., Multivariate Statistical Methods, $4^{\text {a }}$ Ed., 232-320, Editorial Brooks/Cole, New York, USA (2003)

Sayago, Z., M. Chacón y M. Rojas, "Construcción de la identidad profesional docente en estudiantes universitarios", Educere, ISSN: 1316-4910, 12(42), 551-561 (2008)

Schwartzman, G., C. Roni y M. Eder, La formación docente en ciencias de la salud: de recetar a formar, VERTEX Revista Argentina de Psiquiatria, ISSN: 0327-6139, 24, 215-220 (2013)

Stetsenko, A., I.M. Arievitch, The self in Cultural-historical activity theory. Reclaiming the Unity of Social and Individual Dimensions of human development, doi.org/10.177/0959354304044921, Theory \& Psychology, 14(4), 475-503 (2004)

Tejada, F.J., Profesionalización docente en la universidad: implicaciones desde la formación. doi:10.7238/rusc.v10i1.1471, Revista de Universidad y Sociedad del Conocimiento (RUSC), 10(1), 170-184 (2013)

Tenti Fanfani, E., El oficio docente: Vocación, trabajo y profesión en el siglo XXI, 2ª Ed., 180-240, Editorial Siglo XXI, Buenos Aires, Argentina (2005)

Vloet, K. y J. Van Swet, 'I can only learn in dialogue! Exploring profesional identities in teacher education, doi:10.1080/19415250903457083, Journal Professional Development in Education, 36(1-2), 149-168(2010) 
